\title{
Reference Ranges and Determinants of Tricuspid Regurgitation Velocity in Healthy Adults Assessed by Two-Dimensional Doppler-Echocardiography
}

\author{
Alberto M. Marra ${ }^{a}$ Robert Naeije ${ }^{b}$ Francesco Ferrarac ${ }^{c}$ Olga Vriz $^{d}$ \\ Anna Agnese Stanziola ${ }^{e}$ Michele D'Alto ${ }^{f}$ Antonello D'Andrea ${ }^{f}$ \\ Luca Carannante $^{c}$ Christina Alessandra Eichstaedt ${ }^{g}{ }^{h}$ Antonio Cittadini ${ }^{i}$ \\ Nicola Benjaming, h Ekkehard Grünigg, h Eduardo Bossone ${ }^{c}$

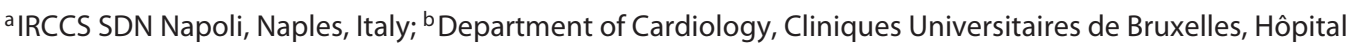 \\ Académique Erasme, Brussels, Belgium; ' Department of Heart, Cardiology Division, "Cava de' Tirreni and Amalfi

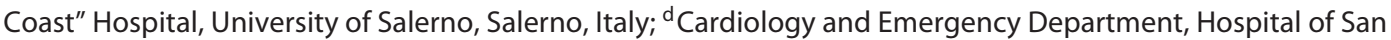 \\ Daniele del Friuli, San Daniele del Friuli, Italy; ${ }^{~}$ Department of Respiratory Diseases, Monaldi Hospital, University

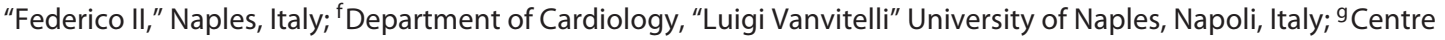 \\ for Pulmonary Hypertension, Thoraxclinic at Heidelberg University Hospital, Heidelberg, Germany; ${ }^{\mathrm{h}}$ German Center \\ of Lung Research (DZL), Giessen, Germany; 'Department of Translational Medical Sciences, University "Federico II", \\ Naples, Italy
}

\section{Keywords}

Tricuspid regurgitation velocity · Healthy subjects .

Doppler-Echocardiography $\cdot$ Reference ranges

\begin{abstract}
Background: Tricuspid regurgitation velocity (TRV) is the most widely used parameter by transthoracic echocardiography (TTE) in the evaluation of patients with suspected pulmonary hypertension (PH). Objectives: To explore the physiologic range of TRV in healthy adults and to investigate its clinical determinants. Methods: In the first part of the study, 614 healthy individuals (age $45.7 \pm 14.7$ years, $60.26 \%$ women) were prospectively assessed by TTE. In the second part, a pooled meta-analysis of 16 already published studies with TRV values in healthy subjects $(n=981)$ was performed. Sta-
\end{abstract}

tistical analysis included the calculation of $95 \%$ quantiles for defining reference ranges. Results: In the prospectively enrolled cohort, mean TRV was $2.01 \pm 0.29 \mathrm{~m} / \mathrm{s}(5-95 \% \mathrm{Cl}$ $1.987-2.033 \mathrm{~m} / \mathrm{s}$ ). It was significantly but weakly positively correlated with age, body mass index, systolic blood pressure, diastolic blood pressure, left atrial volume and negative with mitral inflow E/A ratio. No significant differences were found between males and females. The prospectively enrolled data revealed similar values to the literature-based datasets resulting in a mean TRV of $1.96 \mathrm{~m} / \mathrm{s}(95 \% \mathrm{Cl} 1.91-2.02$ $\mathrm{m} / \mathrm{s}$ ) and a $95 \%$ quantile of $2.55 \mathrm{~m} / \mathrm{s}$. Conclusions: To the best of our knowledge, this is the largest dataset to describe reference ranges of TRV and their clinical determinants in healthy adults years. The determined cut-off value of 2.55 $\mathrm{m} / \mathrm{s}$ of this study has to be confirmed in specific pathologies such as patients at risk of $\mathrm{PH}$. (c) 2018 S. Karger AG, Basel

\section{KARGER}

(c) 2018 S. Karger AG, Basel

E-Mail karger@karger.com

www.karger.com/res
Eduardo Bossone $\mathrm{MD}, \mathrm{PhD}$

Director, Cardiology Division, "Cava de' Tirreni and Amalfi Coast" Hospital Department of Heart, University of Salerno

Via Pr. Amedeo, 36, IT-83023 Lauro (Italy)

E-Mail ebossone@ hotmail.com 


\section{Introduction}

Pulmonary hypertension ( $\mathrm{PH}$ ) occurs in many different diseases and is defined as an increase in mean pulmonary arterial pressure $(\mathrm{PAPm}) \geq 25 \mathrm{~mm} \mathrm{Hg}$ assessed by right heart catheterization (RHC) [1]. Recent data has shown that the normal PAPm at rest is $14+3 \mathrm{~mm} \mathrm{Hg}$ with an upper limit of normal of approximately $20 \mathrm{~mm} \mathrm{Hg} \mathrm{[2].}$ In the new joined guidelines of the European societies for respiratory medicine and cardiology for the diagnosis and treatment of $\mathrm{PH}$, transthoracic color-Doppler-echocardiography (TTE) plays a central role in diagnostic work-up $[1,3,4]$. It should be used to visualize the effects of $\mathrm{PH}$ on the heart and to estimate systolic pulmonary artery pressures through continuous wave Doppler measurements. The European Society of Cardiology (ESC)/ European Respiratory Society (ERS) guidelines recommend grading the probability of $\mathrm{PH}$ based on tricuspid regurgitation velocity (TRV) at rest as high $(\geq 3.5 \mathrm{~m} / \mathrm{s})$, intermediate $(2.9-3.4 \mathrm{~m} / \mathrm{s})$ or low $(\leq 2.8 \mathrm{~m} / \mathrm{s})$, and on the presence of additional echocardiographic variables that suggest $\mathrm{PH}[1]$. Based on these echocardiographic parameters and further clinical findings, a decision must be made about whether RHC is necessary.

Echocardiography should always be performed when $\mathrm{PH}$ is suspected or in $\mathrm{PH}$ patients in follow-up assessments $[1,3,5,6]$. In this regard, TRV is a well-established and most widely used TTE parameter for noninvasive determination of pulmonary pressure [7]. However, the cut-off values proposed in the current ESC/ERS guidelines for establishing the probability of $\mathrm{PH}$ are more or less arbitrary and have not yet been scientifically evaluated or validated for $\mathrm{PH}[3,4,8]$. Retrospective data from a large cohort of patients with high pre-test probability suggests that in experienced hands determining the systolic pulmonary artery pressure using the TRV and estimated right atrial pressure (RAP) correlated well with the invasively measured values $(r=$ 0.87 ) and, when the suggested limit $(36 \mathrm{~mm} \mathrm{Hg}$ ) was used, it has a specificity of $79 \%$ and a sensitivity of $87 \%$ to detect $\mathrm{PH}$ [9]. However, in routine clinical practice, large deviations can occur in individual cases, and only $90 \%$ of patients with $\mathrm{PH}$ had tricuspid regurgitation [10]. Furthermore, the physiologic spectrum of TRV in healthy subjects remains unclear. The aim of this study was to explore the full range of TRV in a large cohort of healthy adults and to perform a meta-analysis of all previously reported normal values, in order to define TRV cut-off values. Furthermore, we want to identify clinical determinants of TRV-values.

\section{Methods}

In the first part of this study, we prospectively assessed consecutive healthy volunteers or healthy subjects being referred for work ability assessment by clinical work-up as previously described [11-13]. Indeed the "Cava de' Tirreni and Amalfi Coast" Hospital is responsible for the assessment of working eligibility for the local area. Examinations included a questionnaire on medical history, use of medications, cardiovascular risk factors and lifestyle habits (alcohol intake, smoking, physical activity). Physical examinations (height, weight, heart rate [HR] and blood pressure $[\mathrm{BP}]$ ) and clinical assessments were conducted according to standardized protocols by trained and certified staff (F.F. and E.B.). Body surface area (BSA) was calculated according to the DuBois formula (BSA $=0.20247 \times$ height $[\mathrm{m}]^{0.725} \times$ weight $\left.[\mathrm{kg}]^{0.425}\right)$. Exclusion criteria were coronary artery disease, systemic arterial hypertension, diabetes mellitus, significant (at least moderate) valvular heart disease, congenital heart disease, congestive heart failure, cardiomyopathies, sinus tachycardia, atrial fibrillation or flutter, use of illicit drugs, elite athletes and inadequate echocardiographic image quality [14]. Three BP measurements were obtained from the right arm by sphygmomanometer and the results were averaged to determine systolic and diastolic BP. Pulse pressure was calculated as the difference between brachial systolic and diastolic BP. None of the subjects had cardiovascular structural or functional abnormalities or received any medication.

In the second part of the study, a systematic literature search and pooled meta-analysis was performed. The study was approved by the institution's ethics board and informed consent was obtained from the participants. The work has been carried out in accordance with the code of Ethics of the World Medical Association (Declaration of Helsinki).

TTE examinations were performed with commercially available equipment (Vivid 7 - GE Healthcare, Milwaukee, WI, USA) as previously described [15]. Specific views included the parasternal long- and short-axis views, apical 4, 2 and 3 chamber $(\mathrm{CH})$ views and subcostal views including respiratory collapse of the inferior vena cava. Pulsed and continuous wave Doppler interrogation was performed on all 4 cardiac valves. Images were recorded digitally and later analysed by 2 independent observers (F.F. and L.C.) using off-line software (EchoPAC - Clinical Workstation Software - GE Healthcare, Milwaukee, WI, USA). All measurements were performed according to the recommendations for echocardiographic assessment of the left and right heart by the American Society of Echocardiography/European Association of Cardiovascular Imaging, available in the enrolment period (June 2007 to February 2014), as previously reported $[12,14,16,17]$.

Taking into account that TRV measurements are angle dependent, TRV was assessed from multiple views, searching for the best envelope and maximal velocity. Peak TRV was measured from the spectral profile of the tricuspid regurgitation jet in the right ventricular $(\mathrm{RV})$ inflow projection of the parasternal longaxis view, the parasternal short-axis view, or the apical 4-chamber view. Care was taken in order to ensure the best alignment between regurgitant flow and the Doppler signal velocity. The highest trans-valvular velocity was used for calculation of TRV [14].

TRV is presented as mean $\pm \mathrm{SD}$. Normal distribution of the continuous values was assessed by the Kolmogorov-Smirnov test. 
Table 1. Demographic characteristics of study population

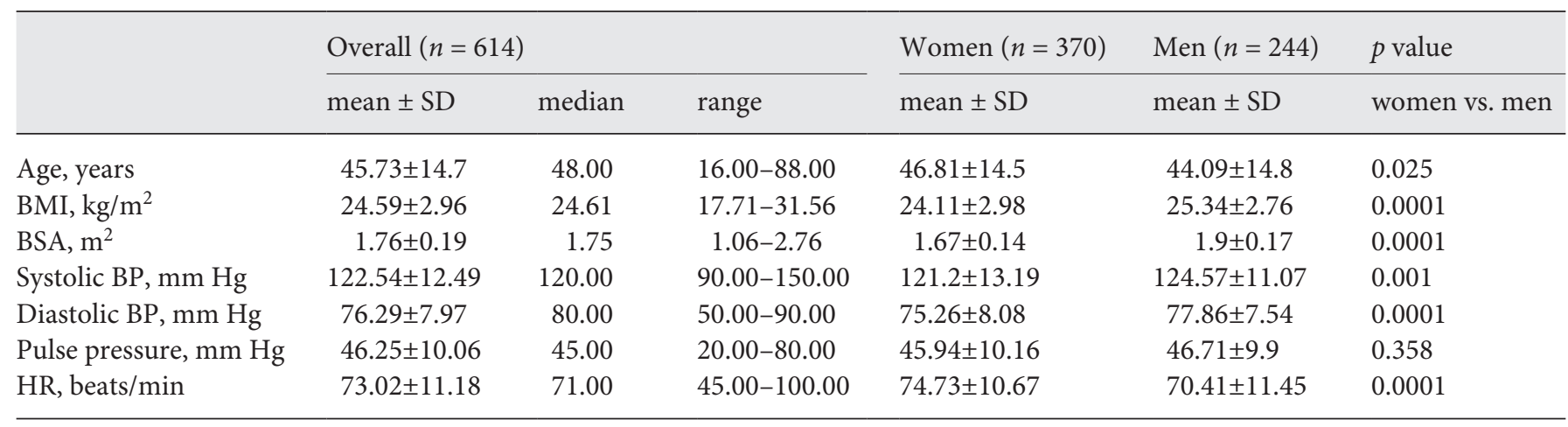

BMI, body mass index; BP, blood pressure; BSA, body surface area; HR, heart rate.

Variables were compared between men and women using the 2 -sided unpaired $t$ test. As upper cut-off values for normal TRV for women and men, we used the $95 \%$ quantile. Partial correlation (Pearson) was used to assess univariate relations. The variables were selected according to their clinical relevance and potential impact on TRV. TRV was also analysed according to age classes $(<30,31-50,51-70$, and $>70$ years $)$ and BMI classes $(<18$, $18-25,25-30$, and $\left.>30 \mathrm{~kg} / \mathrm{m}^{2}\right)$ with ANOVA. Bland-Altman graphs were used to plot the difference between 2 observations versus the mean of the observations to look for overall bias as well as for systematic variation with increased measurement of TRV as described elsewhere [18]. The interobserver variability was examined using both paired $t$ tests and intraclass correlation coefficients (ICCs) in 100 randomly selected cases. An ICC of $>0.8$ indicated good agreement. Analyses were performed by a biometrician (N.B.).

This study was designed according to the Preferred Reporting Items for Systematic reviews and Meta-Analyses statement [19]. A systematic literature search was performed using Medline, Scopus, ISI Web of Sciences and Cochrane Database for studies published between January 1985 and April 2017 reporting echocardiographic TRV-measurements in healthy subjects. Only records in English reporting absolute TRV values were included in the meta-analysis. Studies with unclear echocardiographic techniques or status of subjects were excluded. Studies were identified by the following headings: "right heart," "right ventricle," "tricuspid regurgitation velocity," "systolic pulmonary arterial pressure," "echocardiography," "echocardiogram," "echocardiograms," "Doppler," "ultrasounds" combined with "healthy," "healthy individuals," "healthy subjects," "sane," "control group," "controls." Because only aggregated data, namely, means, SD and sample sizes, are given in the literature, the pooled TRV mean and the pooled SD were used in order to estimate the 95\% percentiles and respective 5-95\% CIs. Mean, 5-95\% CI and $95 \%$ were weighted according to sample size. To analyse the influence of single studies on the pooled estimate, a sensitivity analysis was performed by leaving out one study at a time, calculating the pooled estimates with the remaining studies [13]. All statistical analyses were performed with using SPSS, Version 21 (Chicago, IL, USA).

Reference Ranges and Determinants of Tricuspid Regurgitation Velocity

\section{Results}

\section{Prospective Study}

From June 2007 to March 2017, a sample of 675 healthy subjects was prospectively and consecutively studied by TTE at the Cardiology Division, "Cava de' Tirreni-Amalfi Coast," Heart Department, University Hospital of Salerno, Italy. Among these, 61 subjects (9\%) were excluded due to TRV that was not assessable. The final study cohort consisted of 614 healthy individuals (mean age $45.73 \pm 14.67$ years, range $18-88$ years; 370 [60.26\%] women; Table 1). Compared with men, women were older, had lower body dimensions (BSA and BMI), lower systolic and diastolic BP and higher HRs (Table 1).

Males had higher right and left ventricular (LV) dimensions, as shown by higher values of LV end-diastolic and LV end-systolic volumes, LV mass, and RV diameters. On the other hand, no differences in LV ejection fraction, LV diastolic function and tricuspid annular plane systolic excursion were registered between men and women (Table 2).

TRV showed a normal distribution $(2.00 \pm 0.29 \mathrm{~m} / \mathrm{s}$, $5-95 \%$ CI $1.98-2.03 \mathrm{~m} / \mathrm{s}$, range $1.10-2.85 \mathrm{~m} / \mathrm{s}$; Fig. 1). No differences $(p=0.615)$ were found between males $(2.01 \pm$ $0.31 \mathrm{~m} / \mathrm{s})$ and females $(2.00 \pm 0.27 \mathrm{~m} / \mathrm{s})$. In the univariate analysis, TRV was significantly but weakly positively correlated with systolic BP $(r=0.151, p=0.0001)$, diastolic $\mathrm{BP}(r=0.095, p=0.018)$ and LA volume $(r=0.097, p=$ $0.02)$. TRV correlated negatively and weakly with mitral inflow E/A ratio $(r=-0.109, p=0.007)$. TRV varied significantly according to age classes $(p=0.002$; Fig. 2$)$ and BMI ( $p=0.001$, data not shown). The Bland-Altman analysis (Fig. 3) gave a mean difference of $0.0106 \mathrm{~m} / \mathrm{s}$ (5$95 \%$ CI -0.14 to $0.16 \mathrm{~m} / \mathrm{s}$ ). The interobserver variability 
Table 2. Left and right heart dimension and function

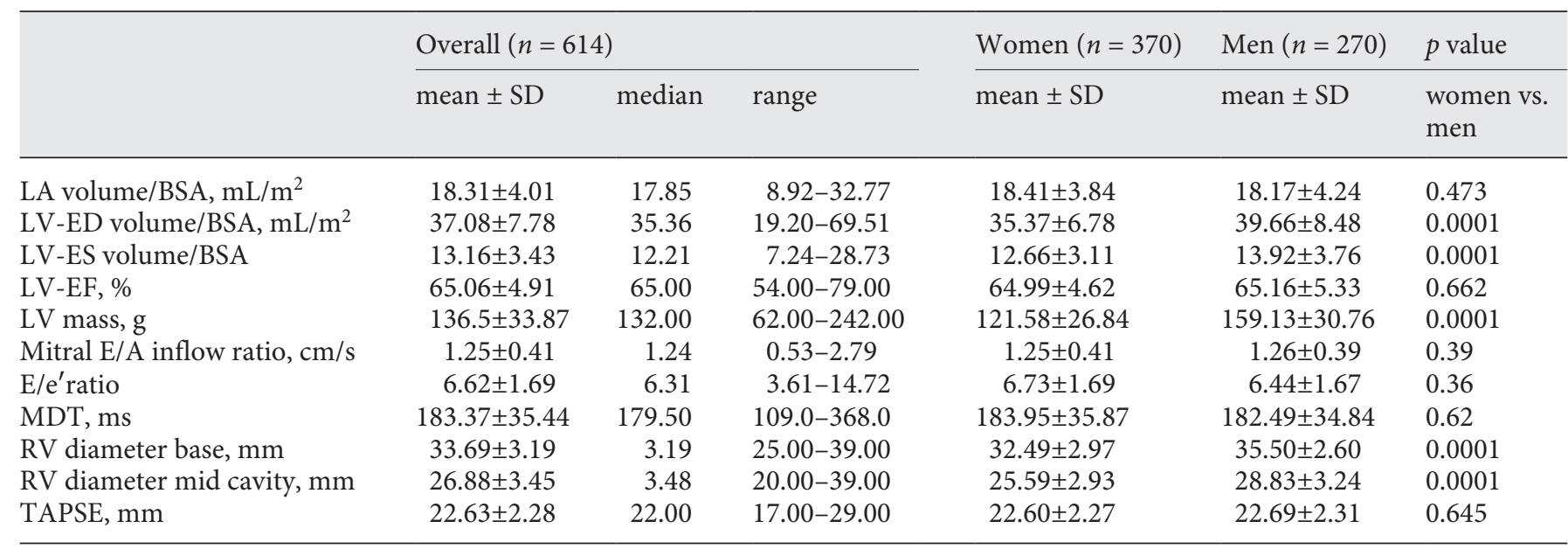

TAPSE, tricuspid annular plane systolic excursion; RV, right ventricle; MDT, mitral deceleration time; LV, left ventricle; EF, ejection fraction; BSA, body surface area; ES, end-systolic; ED, end-diastolic; LA, left atrial.

analysis revealed ICCs of 0.959 for the single measure (5$95 \%$ CI $0.940-0.973)$ and 0.979 for the average of measures (5-95\% CI 0.969-0.989).

\section{Meta-Analysis of Previous Published Studies Pooled with the Prospectively Collected Data}

Table 3 reports the description of the 16 studies found in medical literature reporting TRV values in healthy subjects. Out of 5,389 records detected by screening of the Medline and Cochrane database for TRV using the aforementioned keywords, 90 full-text papers were identified. Seventy-four papers were excluded for several reasons (mainly because the TRV values were not reported). A total number of 16 papers published between 1992 and 2016 was identified (Fig. 4) [10, 20-34]. The pooled analysis of the previously published studies and the current cohort included 1,595 subjects in total. Correlation analyses regarding age and BSA or analyses of differences between sex and training status were not possible because only mean values of TRV were given and no analysis for determinants had been reported in the literature. In all studies, subjects were classified as healthy and free of cardiovascular diseases. Most studies had performed chest $\mathrm{X}$-ray, electrocardiograms and physical examinations to exclude any cardiovascular or respiratory disease. Overall, the assessed subjects were between 18 and 80 years old. The sample size ranged from 14 to 230 subjects per study. All studies, except one [21] included subjects of both sexes. Figure 5 shows the $95 \%$ quantiles and CIs of TRV of previously published studies and our prospective

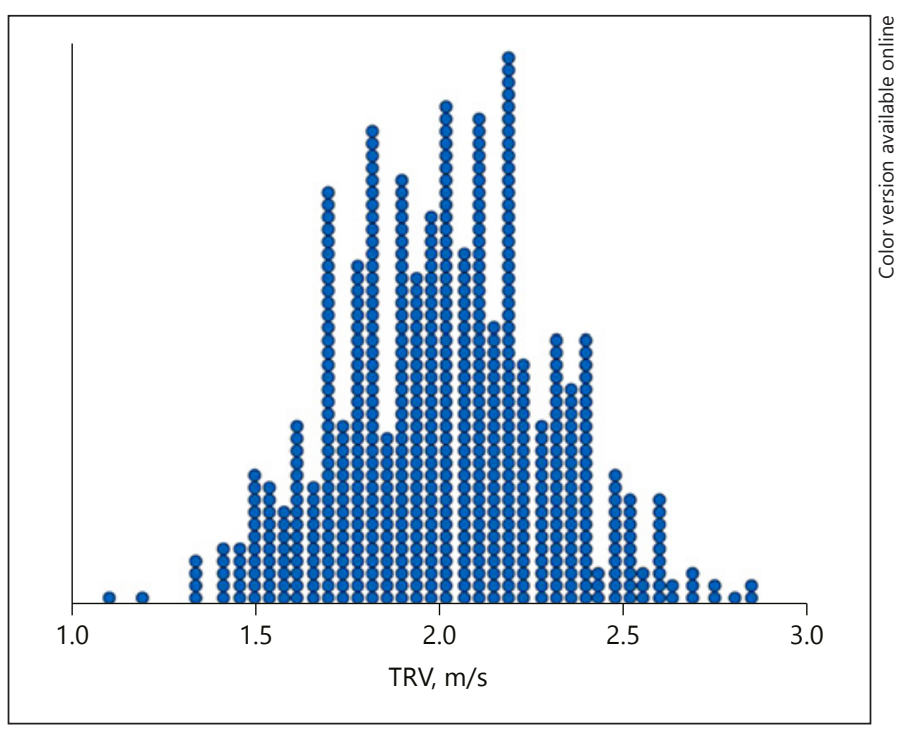

Fig. 1. Distribution of tricuspid regurgitation velocity (TRV) in the prospectively enrolled cohort.

data. Pooled data resulted in a weighted mean TRV of $1.96 \mathrm{~m} / \mathrm{s}(95 \%$ CI $1.90-2.01 \mathrm{~m} / \mathrm{s})$ and a $95 \%$ quantile of $2.55 \mathrm{~m} / \mathrm{s}(95 \%$ CI $1.90-2.01 \mathrm{~m} / \mathrm{s})$.

The influence of each study was analyzed by leaving out one study at a time. If the data of the current study was excluded from the pooled analysis, the cut-off value would be slightly higher ( $95 \%$ quantile: $2.59 \mathrm{~m} / \mathrm{s} ; 95 \%$ CI $1.86-2.02 \mathrm{~m} / \mathrm{s}$ ). The leave-one-out estimates for the $95 \%$ quantiles varied between 2.53 and $2.68 \mathrm{~m} / \mathrm{s}$. Three out of 
614 prospectively enrolled subjects had a TRV measurement higher than $2.8 \mathrm{~m} / \mathrm{s}$, which is the cut-off suggested by current guidelines. Similar results were obtained when excluding these patients (data not shown).

\section{Discussion}

To the best of our knowledge, the current report represents the largest dataset dealing with TRV in healthy individuals. The main finding is that the $95 \%$ quantile of TRV in a healthy population corresponds to $2.55 \mathrm{~m} / \mathrm{s}$, which is lower than the current cut-off of $2.8 \mathrm{~m} / \mathrm{s}$, suggested by the guidelines of $\mathrm{PH}[1]$.

TRV is widely used in daily clinical practice, given its linear positive correlation with sPAP in the absence of RV outflow tract obstruction and/or pulmonic valve stenosis, through the Bernoulli formula $\left(4 \times[\mathrm{TRV}]^{2}+\mathrm{RAP}\right)[3,35$, 36]. The accuracy and the precision of estimation of pulmonary pressures with TTE with regards to $\mathrm{RCH}$ measurements were investigated by D'Alto et al. [7] among 161 patients referred to an echo-lab for the suspicion of $\mathrm{PH}$. According to D'Alto and colleagues, TTE allows accurate measurement of pulmonary pressures (bias and confidence intervals: $\pm 3 \mathrm{~mm} \mathrm{Hg}, \pm 5 \mathrm{~mm} \mathrm{Hg}$, for mPAP and LAP, respectively) but with moderate precision (lower limit mean -SD/upper limit +SD: +19 and $-18 \mathrm{~mm} \mathrm{Hg}$ for $\mathrm{mPAP}$ respectively, and +8 and $-12 \mathrm{~mm} \mathrm{Hg}$ for LAP) leading to the conclusion that the estimation of pulmonary pressure by TTE is valid for population studies but might not be appropriate for the individual diagnosis of $\mathrm{PH}$ [7]. In light of all these aspects, current ESC/ERS joint guidelines on PH [1], as well as American Society of Echocardiography guidelines on right chambers assessment [16], suggest that a TTE be performed whenever a $\mathrm{PH}$ is clinically suspected, due to the utmost importance of an early $\mathrm{PH}$ diagnosis, regardless of its cause. Along with other echocardiographic indexes and clinical signs, guidelines recommend the use of the absolute value of TRV and not the estimated sPAP as main value to confer the echocardiographic probability of $\mathrm{PH}$ [1]. Indeed, they indicate that $\mathrm{PH}$ diagnosis can be excluded with a high likelihood for patients with a TRV less than $2.8 \mathrm{~m} / \mathrm{s}$ [1]. Kovacs et al. [2] performed a comprehensive systematic review on 1,187 individuals from 47 studies in 13 countries in order to determine the normal values of pulmonary pressures assessed by RHC. In this study, an upper limit of normality of $29.6 \mathrm{mmHg}$ was found for sPAP. According to Bernoulli formula and assuming a RAP of $5 \mathrm{~mm} \mathrm{Hg}$, the upper limit of normality suggested by our

Reference Ranges and Determinants of Tricuspid Regurgitation Velocity

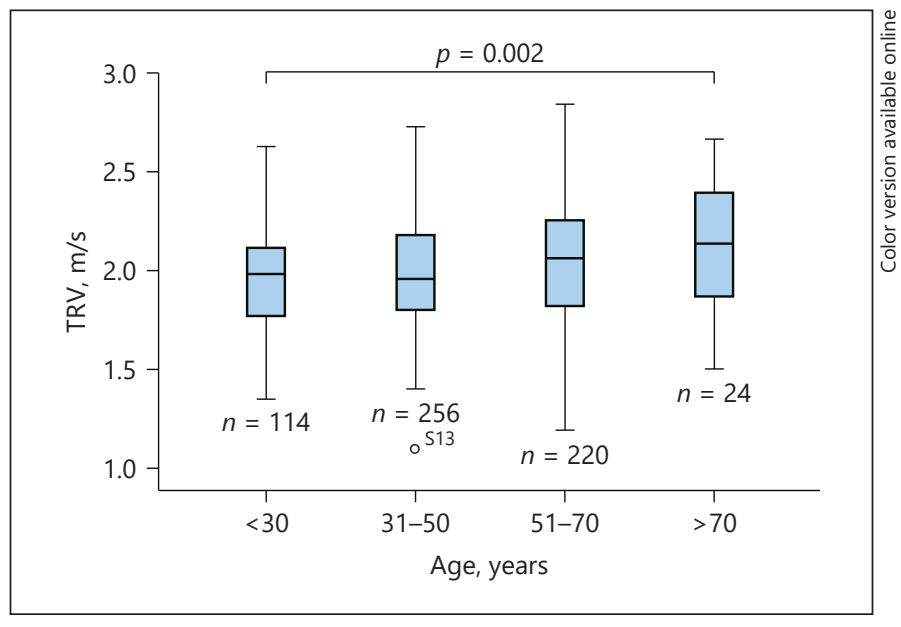

Fig. 2. Tricuspid regurgitation velocity (TRV) according to different age classes.

current report $(2.55 \mathrm{~m} / \mathrm{s})$ would lead to an estimated sPAP of $31.01 \mathrm{~mm} \mathrm{Hg}$, an upper limit very close to $29.6 \mathrm{~mm} \mathrm{Hg}$ reported by Kovacs et al. [2]. Interestingly, our results are in line with the results of a previous prospective study from Grünig et al. [13] assessing 191 healthy controls [22] and identifying a TRV at rest of $1.96 \pm 0.25 \mathrm{~m} / \mathrm{s}$, which was not correlated with gender [22]. McQuillan et al. [37] evaluated TRV retrospectively from a database not explicitly excluding patients with coronary artery disease and/or pulmonary diseases. In this study, 95\% upper limit was equivalent to a TRV of $2.61 \mathrm{~m} / \mathrm{s}$. However, the higher values may be due to a different cohort as in-hospital patients were assessed.

In our prospectively enrolled cohort, TRV was very weakly positively correlated with age and BMI. The linear relation between cardiac output and BMI is well established, leading to the speculation that subjects with higher body mass have higher cardiac output and consequently slightly higher pulmonary pressures [38]. However, our data and a previous prospective study [22] do not support this assumption, given the low correlation between BMI and TRV that we found. Indeed, only $2.5 \%$ of the TRV data might be explained by age.

Other indices weakly correlating with TRV were arterial BP, LA volume and diastolic function. All these parameters are associated with a backward transmission of pressure from the left ventricle to the pulmonary circulation [39]. Although right heart dimensions in healthy adults vary in the 2 sexes $[13,15]$, with men having larger RV [13] and RA [15] than women, pulmonary pressure values are similar in men and women in physiologic states [2], albeit women are more prone to develop PH 
Fig. 3. Bland Altman plot of tricuspid regurgitation velocity (TRV) assessment between the two operators. The red thick line represents mean difference and the red thin lines represent $95 \%$ of the confidence interval. The blue thin lines represent the limits of agreement (5-95\% CI).

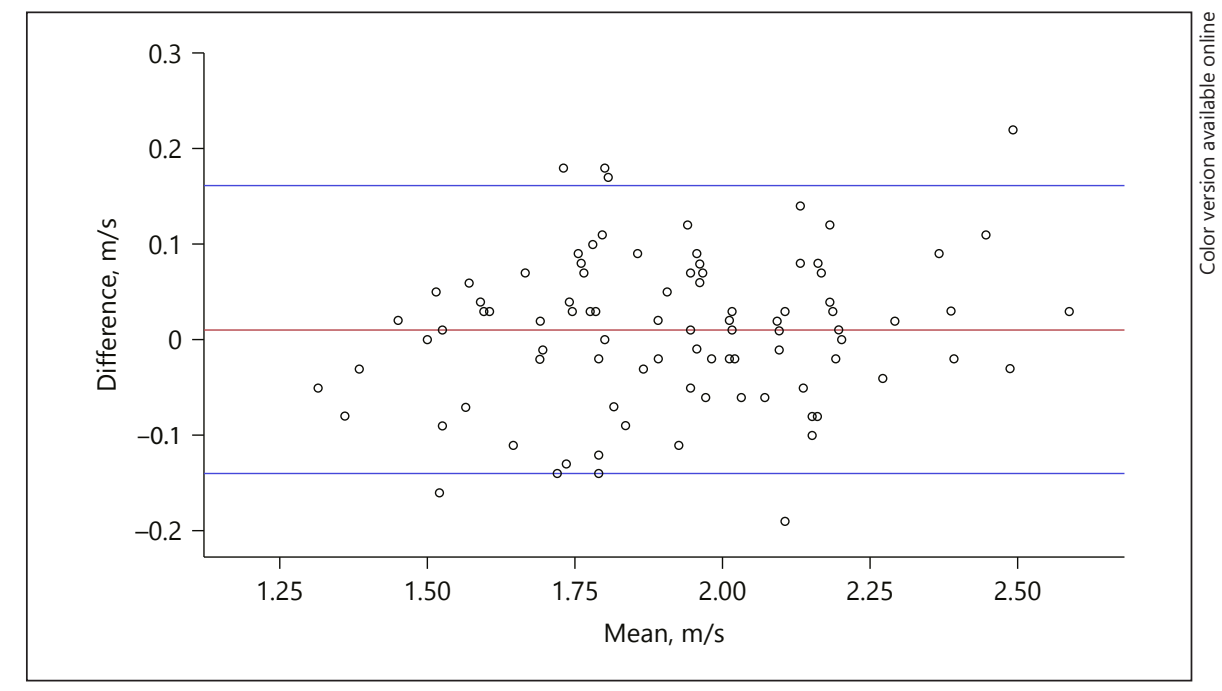

Table 3. Pooled studies reporting TRV values in healthy subjects

\begin{tabular}{|c|c|c|c|c|c|c|c|c|c|}
\hline Author & Year & Country & Setting & $\begin{array}{l}\text { Sample } \\
n\end{array}$ & $\begin{array}{l}\text { Females } \\
n\end{array}$ & $\begin{array}{l}\text { Age, years } \\
\text { mean } \pm S D\end{array}$ & $\begin{array}{l}\mathrm{TRV}, \mathrm{m} / \mathrm{s} \\
\text { mean } \pm \mathrm{SD}\end{array}$ & $\begin{array}{l}\mathrm{TRV}, \mathrm{m} / \mathrm{s} \\
95 \% \mathrm{CI}\end{array}$ & $\begin{array}{l}\text { TRV }, \mathrm{m} / \mathrm{s} \\
5-95 \% \\
\text { quantile }\end{array}$ \\
\hline Bossone [15] & 1999 & USA & Control vs. Athletes & 14 & 0 & $18.0 \pm 0.9$ & $1.70 \pm 0.11$ & $1.64-1.75$ & $1.51-1.88$ \\
\hline Aessopos [16] & 2000 & Greece & Healthy volunteers & 53 & 34 & $38.9 \pm 12.7$ & $2.10 \pm 0.23$ & $2.03-2.16$ & $1.72-2.47$ \\
\hline Bossone [17] & 2000 & USA & Controls vs. PH & 22 & 12 & $38.4 \pm 8.3$ & $2.30 \pm 0.25$ & $2.19-2.40$ & $1.88-2.71$ \\
\hline Mahjoub [20] & 2009 & France & Healthy volunteers & 70 & 36 & $48.0 \pm 16.0$ & $2.00 \pm 0.20$ & $1.95-2.04$ & $1.67-2.32$ \\
\hline Sahin [21] & 2009 & Turkey & Controls vs. HT & 30 & 22 & $47.4 \pm 10.5$ & $2.05 \pm 0.30$ & $1.94-2.15$ & $1.55-2.54$ \\
\hline Grünig [22] & 2009 & Germany & $\begin{array}{l}\text { Controls vs. relatives } \\
\text { of IPAH and HPAH }\end{array}$ & 191 & 78 & $37.0 \pm 16.0$ & $1.96 \pm 0.25$ & $1.92-1.99$ & $1.54-2.37$ \\
\hline Celik [23] & 2011 & Turkey & Controls vs. PTE & 20 & 11 & $56.0 \pm 7.0$ & $1.70 \pm 0.30$ & $1.56-1.83$ & $1.20-2.19$ \\
\hline Grapsa [24] & 2012 & UK & Controls vs. PAH & 30 & 22 & $\mathrm{nr} \pm \mathrm{nr}$ & $1.60 \pm 0.30$ & $1.49-1.70$ & $1.10-2.09$ \\
\hline Lin [29] & 2016 & China & Controls vs. CP & 43 & 24 & $46.8 \pm 14.5$ & $2.13 \pm 0.30$ & $2.04-2.22$ & $1.63-2.62$ \\
\hline Current study & 2017 & Italy & Healthy volunteers & 614 & 370 & $45.7 \pm 14.7$ & $2.01 \pm 0.29$ & $1.98-2.03$ & $1.53-2.48$ \\
\hline Pooled & - & - & - & 1,595 & 825 & - & $1.96^{*}$ & $1.90-2.01^{*}$ & $1.37-2.55^{*}$ \\
\hline
\end{tabular}

TRV, tricuspid regurgitation velocity; $\mathrm{PH}$, pulmonary hypertension; SCD, sickle cells disease; LBBB, left bundle branch block; ex- $\mathrm{PH}$, exercise pulmonary hypertension; HAT, hashimoto's thyroiditis; IPAH, idiopathic pulmonary arterial hypertension; HPAH, heritable pulmonary arterial hypertension; PTE, pulmonary thromboembolism; PAH, pulmonary arterial hypertension; HTR, heart transplant recipients; $\mathrm{CP}$, constrictive pericarditis; nr, not reported. * Weighted.

[40]. This might lead to the speculation that right heart chambers are more dependent from body size than TRV. Based on all these conclusions, we might assume that TRV is a stable index, being weakly influenced by other parameters and variables. This is also true taking into ac- count the minimal intra-observer and inter-observer variability we recorded (Fig. 3). Our study owes few limitations.

First, correlations between TTE data and invasively assessed pulmonary pressures were not investigated. How- 
Fig. 4. PRISMA 2009 flow diagram. TRV, tricuspid regurgitation velocity.

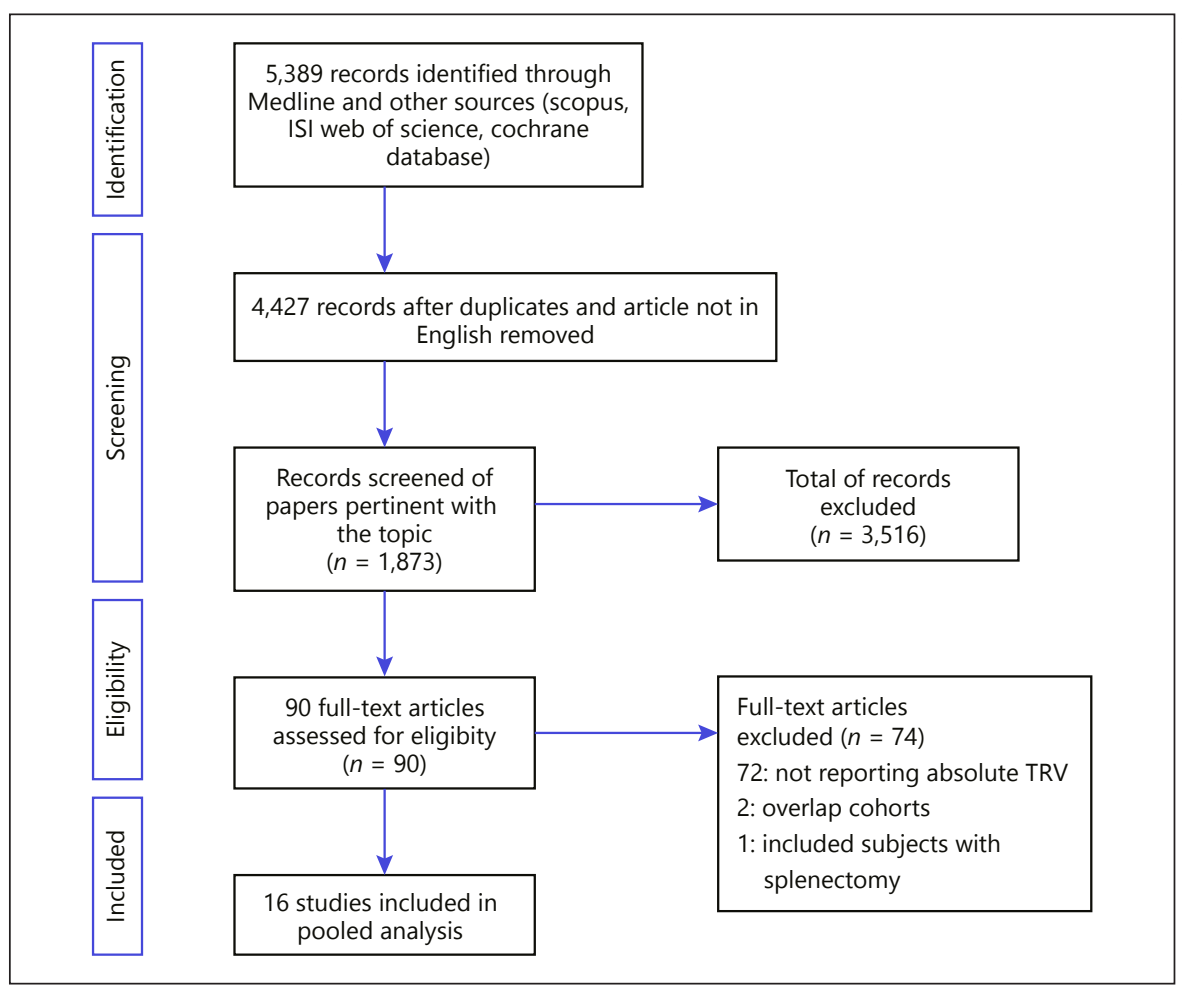

Fig. 5. Forest plot of pooled data regarding tricuspid regurgitation velocity (TRV) in healthy subjects. The forest plot presents the $95 \%$ quantile of the TRV and the range of $95 \%$ confidence intervals of our prospective data and previous publications selected for pooled analysis. The bigger value at the bottom of the graph shows the weighted pooled confidence interval for the $95 \%$ quantile of TRV $(2.55 \mathrm{~m} / \mathrm{s})$.

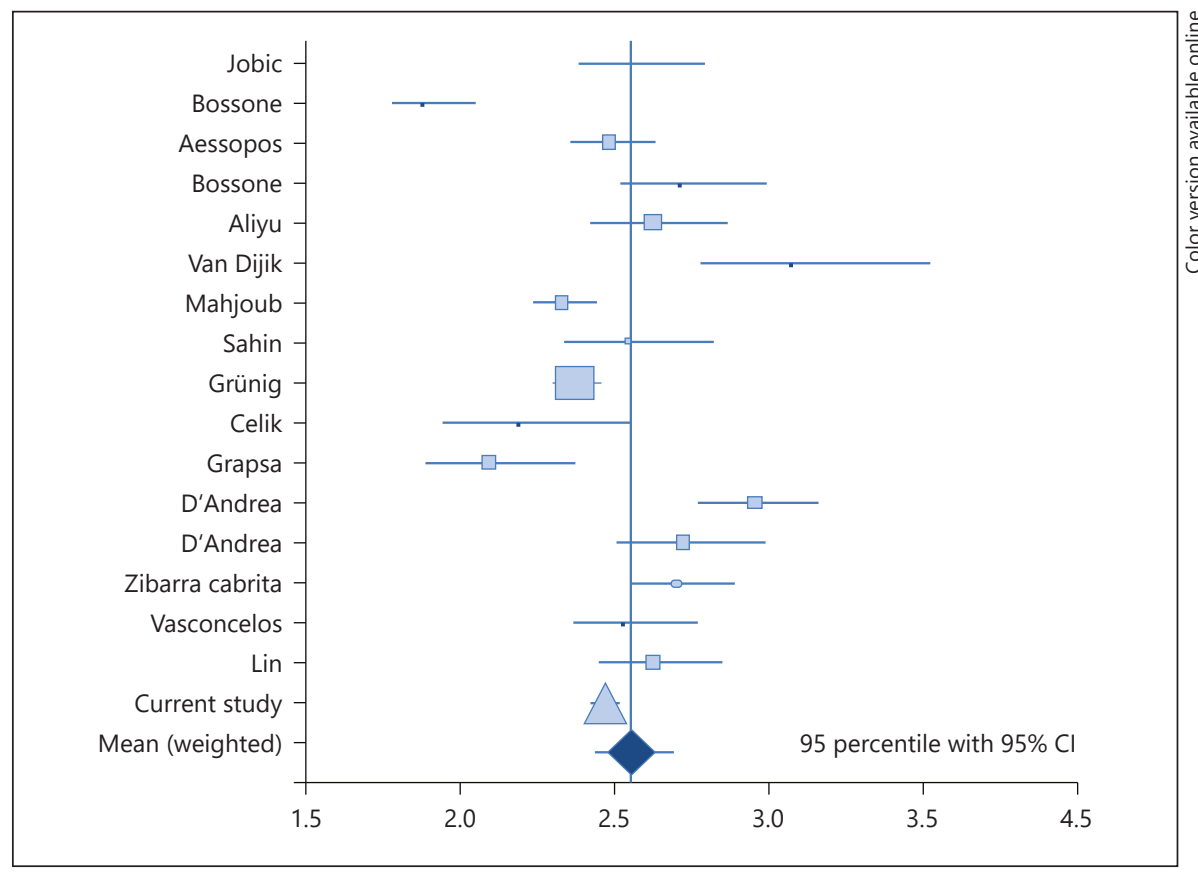

ever, it would be unethical to perform such an invasive procedure in healthy individuals without a clinical indication. Second, our sample did not include severely obese individuals, with only 22 patients among 614 (3.6\%) mildly obese, with a BMI $>30 \mathrm{~kg} / \mathrm{m}^{2}$ with a highest value of BMI of $31.56 \mathrm{~kg} / \mathrm{m}^{2}$. However, similar results would have been obtained, if these 22 subjects with a BMI above $30 \mathrm{~kg} / \mathrm{m}^{2}$ had been excluded from the analysis (mean TRV $1.95 \mathrm{~m} / \mathrm{s} ; 95 \%$ quantile $2.57 \mathrm{~m} / \mathrm{s}$ ). The $95 \%$ quantile was used as normal reference values because the largest 
$5 \%$ of values may indicate pathological processes. However, if a $97.5 \%$ percentile were used, the reference values would change only slightly $(2.66 \mathrm{~m} / \mathrm{s})$.

In conclusion, the current report provides the resting upper physiologic limits of TRV and may serve as a further insight in understanding non-invasive assessment of pulmonary pressure under physiologic circumstances. The meaning of a cut-off of $2.55 \mathrm{~m} / \mathrm{s}$ for the risk evaluation of $\mathrm{PH}$ should be further investigated, as it might help to identify more subjects who are prone to develop or al- ready have a manifest $\mathrm{PH}$. Moreover, according to our data, TRV is a robust and stable index, without any clinical meaningful influence from body size, age, sex and it is characterized by a strong reproducibility.

\section{Disclosure Statement}

The authors declare that they have no conflicts of interest to disclose.

\section{References}

1 Galiè N, Humbert M, Vachiery JL, Gibbs S, Lang I, Torbicki A, et al: 2015 ESC/ERS Guidelines for the diagnosis and treatment of pulmonary hypertension. Eur Heart J 2015; 46:ehv317.

2 Kovacs G, Berghold A, Scheidl S, Olschewski $\mathrm{H}$ : Pulmonary arterial pressure during rest and exercise in healthy subjects: a systematic review. Eur Respir J 2009;34:888-894.

3 Bossone E, Dellegrottaglie S, Patel S, Grunig E, D'Andrea A, Ferrara F, et al: Multimodality imaging in pulmonary hypertension. Can J Cardiol 2015;31:440-459.

4 Kovacs G, Dumitrescu D, Barner A, Greiner S, Grünig E, Hager A, et al: Klinische Klassifikation der pulmonalen Hypertonie und initiale Diagnostik: Empfehlungen der Kölner Konsensus Konferenz 2016. Dtsch Medizinische Wochenschrift 2016;141:S10-S18.

5 Bossone E, D'Andrea A, D'Alto M, Citro R, Argiento P, Ferrara F, et al: Echocardiography in pulmonary arterial hypertension: from diagnosis to prognosis. J Am Soc Echocardiogr 2013;26:1-14.

6 Ferrara F, Gargani L, Ostenfeld E, D’Alto M, Kasprzak J, Voilliot D, et al: Imaging the right heart pulmonary circulation unit: Insights from advanced ultrasound techniques. Echocardiography 2017;34:1216-1231.

7 D'Alto M, Romeo E, Argiento P, D'Andrea A, Vanderpool R, Correra A, et al: Accuracy and precision of echocardiography versus right heart catheterization for the assessment of pulmonary hypertension. Int J Cardiol 2013; 168:4058-4062.

8 Lewis GD, Murphy RM, Shah RV, Pappagianopoulos PP, Malhotra R, Bloch KD, et al: Pulmonary vascular response patterns during exercise in left ventricular systolic dysfunction predict exercise capacity and outcomes. Circ Heart Fail 2011:4:276-285.

9 Greiner S, Jud A, Aurich M, Hess A, Hilbel T, Hardt S, et al: Reliability of noninvasive assessment of systolic pulmonary artery pressure by Doppler echocardiography compared to right heart catheterization: analysis in a large patient population. J Am Heart Assoc 2014;3:e01103-e001103.
10 Grünig E, Weissmann S, Ehlken N, Fijalkowska A, Fischer C, Fourme T, et al: Stress Doppler echocardiography in relatives of patients with idiopathic and familial pulmonary arterial hypertension: results of a multicenter European analysis of pulmonary artery pressure response to exercise and hypoxia. Circulation 2009;119:1747-1757.

11 Ferrara F, Rudski LG, Vriz O, Gargani L, Afilalo J, D’Andrea A, et al: Physiologic correlates of tricuspid annular plane systolic excursion in 1168 healthy subjects. Int J Cardiol 2016;223:736-743.

12 Marra AM, Benjamin N, Ferrara F, Vriz O, D'Alto M, D'Andrea A, et al: Reference ranges and determinants of right ventricle outflow tract acceleration time in healthy adults by two-dimensional echocardiography. Int J Cardiovasc Imaging 2017;33:219-226.

13 Grünig E, Biskupek J, D’Andrea A, Ehlken N, Egenlauf B, Weidenhammer J, et al: Reference ranges for and determinants of right ventricular area in healthy adults by two-dimensional echocardiography. Respiration 2015;89:284293.

14 Ferrara F, Rudski LG, Vriz O, Gargani L, Afilalo J, D'Andrea A, et al: Physiologic correlates of tricuspid annular plane systolic excursion in 1168 healthy subjects. Int J Cardiol 2016;223:736-743.

15 Grunig E, Henn P, D'Andrea A, Claussen M, Ehlken N, Maier F, et al: Reference values for and determinants of right atrial area in healthy adults by 2-dimensional echocardiography. Circ Cardiovasc Imaging 2013;6:117124.

16 Rudski LG, Lai WW, Afilalo J, Hua L, Handschumacher MD, Chandrasekaran $\mathrm{K}$, et al: Guidelines for the echocardiographic assessment of the right heart in adults: a report from the American Society of Echocardiography endorsed by the European Association of Echocardiography, a registered branch of the European Society of Cardiology, and the Canadian Society of Echocardiography. J Am Soc Echocardiogr 2010;23:685-713.

17 Lang RM, Badano LP, Mor-Avi V, Afilalo J, Armstrong A, Ernande L, et al: Recommenda- tions for cardiac chamber quantification by echocardiography in adults: an update from the American Society of Echocardiography and the European Association of Cardiovascular Imaging. J Am Soc Echocardiogr 2015; 28:1-39.e14.

18 Marra AM, Egenlauf B, Ehlken N, Fischer C, Eichstaedt C, Nagel C, Bossone E, Cittadini A, Halank M, Gall H, Olsson KM, Lange TJ, et al: Change of right heart size and function by long-term therapy with riociguat in patients with pulmonary arterial hypertension and chronic thromboembolic pulmonary hypertension. Int J Cardiol 2015;195:19-26.

19 Moher D, Liberati A, Tetzlaff J, Altman DG PRISMA Group: Preferred reporting items for systematic reviews and meta-analyses: the PRISMA statement. PLoS Med 2009;6: e1000097.

20 Jobic Y, Slama M, Tribouilloy C, Lan Cheong Wah L, Choquet D, Boschat J, et al: Doppler echocardiographic evaluation of valve regurgitation in healthy volunteers. Br Heart J 1993;69:109-113.

21 Bossone E, Rubenfire M, Bach DS, Ricciardi M, Armstrong WF: Range of tricuspid regurgitation velocity at rest and during exercise in normal adult men: implications for the diagnosis of pulmonary hypertension. J Am Coll Cardiol 1999;33:1662-1666.

22 Aessopos A, Farmakis D, Taktikou H, Loukopoulos D: Doppler-determined peak systolic tricuspid pressure gradient in persons with normal pulmonary function and tricuspid regurgitation. J Am Soc Echocardiogr 2000;13: 645-649.

23 Bossone E, Avelar E, Bach DS, Gillespie B, Rubenfire M, Armstrong WF: Diagnostic value of resting tricuspid regurgitation velocity and right ventricular ejection flow parameters for the detection of exercise induced pulmonary arterial hypertension. Int J Card Imaging 2000;16:429-436.

24 Aliyu ZYY Gordeuk V, Sachdev V, Babadoko A, Mamman AI, Akpanpe P, et al: Prevalence and risk factors for pulmonary artery systolic hypertension among sickle cell disease patients in Nigeria. Am J Hematol 2008;83:485-490. 
25 van Dijk J, Knaapen P, Bekkering I, Gotte MJ, Kamp O: Right ventricular dimensions and function in isolated left bundle branch block: is there evidence of biventricular involvement? Echocardiography 2008;25:457464.

26 Mahjoub H, Levy F, Cassol M, Meimoun P, Peltier M, Rusinaru D, et al: Effects of age on pulmonary artery systolic pressure at rest and during exercise in normal adults. Eur J Echocardiogr 2009; 10:635-640.

27 Sahin M, Sade LE, Tutuncu NB, Gursoy A, Kebapcilar L, Muderrisoglu H, et al: Systolic pulmonary artery pressure and echocardiographic measurements in patients with euthyroid Hashimoto's thyroiditis. J Endocrinol Invest 2009;32:530-532.

28 Celik A, Kocyigit I, Calapkorur B, Korkmaz H, Doganay E, Elcik D, et al: Tenascin-C may be a predictor of acute pulmonary thromboembolism. J Atheroscler Thromb 2011;18:487893.

29 Grapsa J, Gibbs JS, Cabrita IZ, Watson GF, Pavlopoulos H, Dawson D, et al: The association of clinical outcome with right atrial and ventricular remodelling in patients with pulmonary arterial hypertension: study with real-time three-dimensional echocardiography.
Eur Hear J Cardiovasc Imaging 2012;13:666672.

30 D'Andrea A, Riegler L, Golia E, Cocchia R, Scarafile R, Salerno G, et al: Range of right heart measurements in top-level athletes: the training impact. Int J Cardiol 2013;164:48-57.

31 D'Andrea A, Riegler L, Nunziata L, Scarafile R, Gravino R, Salerno G, et al: Right heart morphology and function in heart transplantation recipients. J Cardiovasc Med 2013;14:648-658.

32 Zimbarra Cabrita I, Ruísanchez C, Grapsa J, Dawson D, North B, Pinto FJ, et al: Validation of the isovolumetric relaxation time for the estimation of pulmonary systolic arterial blood pressure in chronic pulmonary hypertension. Eur Heart J Cardiovasc Imaging 2013; 14:51-55.

33 Vasconcelos MC, Nunes MC, Barbosa MM, Fernandes BM, Passaglia LG, Silva CM, et al: Left ventricular remodeling in patients with sickle cell disease: determinants factors and impact on outcome. Ann Hematol 2015;94: 1621-1629.

34 Lin X, Xu RY, Liu JZ, Chen W, Chen LF, Yang $\mathrm{PH}$, et al: Effect of right heart systolic function on outcomes in patients with constrictive pericarditis undergoing pericardiectomy. Chin Med J (Engl) 2016;129:154.
35 Yock PG, Popp RL: Noninvasive estimation of right ventricular systolic pressure by Doppler ultrasound in patients with tricuspid regurgitation. Circulation 1984;70:657662.

36 Bossone E, Ferrara F, Grünig E: Echocardiography in pulmonary hypertension. Curr Opin Cardiol 2015;30:574-586.

37 McQuillan BM, Picard MH, Leavitt M, Weyman AE: Clinical correlates and reference intervals for pulmonary artery systolic pressure among echocardiographically normal subjects. Circulation 2001;104:2797-2802.

38 D'Andrea A, Naeije R, Grünig E, Caso P, D’Alto M, Di Palma E, et al: Echocardiography of the pulmonary circulation and right ventricular function: exploring the physiologic spectrum in 1,480 normal subjects. Chest 2014;145:1071-1078.

39 Rosenkranz S, Gibbs JS, Wachter R, De Marco T, Vonk-Noordegraaf A, Vachiéry JL: Left ventricular heart failure and pulmonary hypertension. Eur Heart J 2016;37:942-954.

40 Marra AM, Benjamin N, Eichstaedt C, Salzano A, Arcopinto M, Gargani L, et al: Genderrelated differences in pulmonary arterial hypertension targeted drugs administration. Pharmacol Res 2016;114:103-109. 